\title{
Rosetta and Mars Express observations of the influence of high solar wind pressure on the Martian plasma environment
}

\author{
N. J. T. Edberg ${ }^{1,9}$, U. Auster ${ }^{2}$, S. Barabash ${ }^{3}$, A. Bößwetter ${ }^{4}$, D. A. Brain ${ }^{5}$, J. L. Burch ${ }^{6}$, C. M. Carr ${ }^{7}$, S. W. H. Cowley ${ }^{1}$, \\ E. Cupido ${ }^{7}$, F. Duru ${ }^{8}$, A. I. Eriksson 9 , M. Fränz ${ }^{10}$, K.-H. Glassmeier ${ }^{2,10}$, R. Goldstein ${ }^{6}$, M. Lester ${ }^{1}$, R. Lundin ${ }^{3}$, \\ R. Modolo ${ }^{11}$, H. Nilsson ${ }^{3}$, I. Richter ${ }^{2}$, M. Samara ${ }^{6}$, and J. G. Trotignon ${ }^{12}$ \\ ${ }^{1}$ Department of Physics \& Astronomy, University of Leicester, Leicester LE1 7RH, UK \\ ${ }^{2}$ Institute for Geophysics and Extraterrestrial Physics, TU Braunschweig, Germany \\ ${ }^{3}$ Swedish Institute of Space Physics, Kiruna, Sweden \\ ${ }^{4}$ Institute for Theoretical Physics, TU Braunschweig, Germany \\ ${ }^{5}$ Space Sciences Laboratory, University of California, Berkeley, USA \\ ${ }^{6}$ Southwest Research Institute, San Antonio, USA \\ ${ }^{7}$ The Blackett Laboratory, Imperial College London, London, UK \\ ${ }^{8}$ Department of Physics and Astronomy, University of Iowa, Iowa City, USA \\ ${ }^{9}$ Swedish Institute of Space Physics, Uppsala, Sweden \\ ${ }^{10}$ Max Planck Institute for Solar System Research, Katlenburg-Lindau, Germany \\ ${ }^{11}$ CETP-IPSL, 10-12 avenue de l'Europe, 78140 Velizy, France \\ ${ }^{12}$ Laboratoire de Physique et Chimie de l'Environnement, Orléans, France
}

Received: 16 June 2009 - Revised: 26 October 2009 - Accepted: 7 December 2009 - Published: 17 December 2009

\begin{abstract}
We report on new simultaneous in-situ observations at Mars from Rosetta and Mars Express (MEX) on how the Martian plasma environment is affected by high pressure solar wind. A significant sharp increase in solar wind density, magnetic field strength and turbulence followed by a gradual increase in solar wind velocity is observed during $\sim 24 \mathrm{~h}$ in the combined data set from both spacecraft after Rosetta's closest approach to Mars on 25 February 2007. The bow shock and magnetic pileup boundary are coincidently observed by MEX to become asymmetric in their shapes. The fortunate orbit of MEX at this time allows a study of the inbound boundary crossings on one side of the planet and the outbound crossings on almost the opposite side, both very close to the terminator plane. The solar wind and interplanetary magnetic field (IMF) downstream of Mars are monitored through simultaneous measurements provided by Rosetta. Possible explanations for the asymmetries are discussed, such as crustal magnetic fields and IMF direction. In the same interval, during the high solar wind pressure pulse, MEX observations show an increased amount of escaping planetary ions from the polar region of Mars. We link the high pressure solar wind with the observed simultaneous ion
\end{abstract}

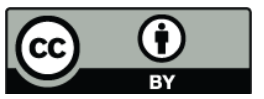

Correspondence to: N. J. T. Edberg (ne@irfu.se) outflow and discuss how the pressure pulse could also be associated with the observed boundary shape asymmetry.

Keywords. Interplanetary physics (Planetary bow shocks) Magnetospheric physics (Magnetosheath; Solar wind interactions with unmagnetized bodies)

\section{Introduction}

Rosetta was launched in 2004 and performed a swingby of Mars on 25 February 2007 on its way to comet 67P/Churyumov-Gerasimenko (Glassmeier et al., 2007a). The Rosetta Mars swingby then enabled a two-spacecraft multi-instrument study of the Martian plasma environment together with Mars Express (MEX) (Edberg et al., 2009b; Boesswetter et al., 2009).

The solar wind interaction with Mars forms the bow shock (BS) where the supersonically flowing solar wind slows down and becomes heated and more turbulent. At altitudes below the BS a thick magnetosheath is characterised by heated and turbulent plasma. The magnetosheath stretches down to the magnetic pileup boundary (MPB) where interplanetary magnetic field (IMF) piles up and drapes around the planet. Factors controlling the location of the BS and MPB include the IMF direction (Zhang et al., 1991; Vignes et al., 2002; Brain et al., 2005; Dubinin et al., 2008a), crustal

Published by Copernicus Publications on behalf of the European Geosciences Union. 
fields (Crider et al., 2002; Edberg et al., 2008; Dubinin et al., 2008b) and the solar wind dynamic pressure (Crider et al., 2003; Brain et al., 2005; Dubinin et al., 2006). They have all been shown to be important for determining the location of the boundaries in statistical studies. Dubinin et al. (2006) performed a large study using data from 400 orbits of MEX during its first year at Mars to study the influence of all of the above factors, and also including the solar EUV flux, on the MPB. Edberg et al. (2009a) have performed an extended study in relation to that, using the first 5 years of measurements to perform a similar study, and also included the BS, in which explicit expressions for the altitude of the boundaries as functions of the above factors were derived. However, for individual observations of the boundaries these factors all contribute and mix with each other making the interaction more complicated.

The shape and location of the Martian BS and MPB have been studied extensively in the past (Vignes et al., 2000; Dubinin et al., 2006; Edberg et al., 2008, and references therein). In the Mars solar orbital (MSO) system, where the $\mathrm{x}$-axis is directed toward the Sun, the z-axis is directed along the Mars orbital angular momentum vector and the $y$-axis completes the right-handed system, both the BS and the MPB have the average shape of conic sections $r=L /(1+\epsilon \cos (\theta))$, where $r$ and $\theta$ are polar coordinates with origin at $X_{0}$ referenced to the $\mathrm{x}$-axis, $\epsilon$ is the eccentricity and $L$ is the semi-latus rectum. Typical values of $X_{0}, \epsilon$ and $L$ are $0.86 R_{\mathrm{M}}, 0.92$ and $0.90 R_{\mathrm{M}}$ for the MPB and $0.55 R_{\mathrm{M}}, 1.05$ and $2.10 R_{\mathrm{M}}$ for the BS, respectively (Edberg et al., 2008) $\left(1 R_{\mathrm{M}}=3397 \mathrm{~km}\right)$.

Dubinin et al. (2009) studied an event where a corotating interaction region (CIR) impacts on Mars and the effect of this is shown to be a scavenged ionosphere, meaning that the high pressure solar wind plasma penetrates the MPB and reaches the ionosphere where it sweeps out large portions of ionospheric plasma. The penetrating solar wind opens up several escape channels for the planetary plasma which leads to a significantly lower ionospheric plasma density (Dubinin et al., 2009). Nilsson et al. (2009) have also performed a statistical study showing that the outflow of ions increases with increased solar wind dynamic pressure.

Pérez-de-Tejada et al. (2009) performed a study where they observe escaping planetary ions on the 25 February 2007 in MEX data. Simultaneous XMM/Newton X-ray images show evidence of an extended and asymmetric exosphere of Mars. Note that these observations took place on the same day as Rosetta had its closest approach to Mars. We can therefore extend their study by using Rosetta for solar wind monitoring and IMF measurements, while MEX at the same time samples the plasma boundaries. There is no magnetometer on MEX, but there is one on Rosetta, such that the swingby of Rosetta provided a unique opportunity of having solar wind magnetic field measurements. MEX can also provide sporadic monitoring of the solar wind during the intervals spent outside the BS. Not reported in the study by Pérez-de-Tejada et al. (2009) is that their observations take place during a period of very active solar wind of high pressure. In this paper we observe asymmetric shapes of the BS and MPB and link this to the outflowing ions and asymmetric exosphere reported by Pérez-de-Tejada et al. (2009) by arguing that the same effect took place as reported by Dubinin et al. (2009) during a high pressure CIR.

\section{Mars Express and Rosetta observations}

Rosetta is equipped with a suite of five plasma instruments out of which three are used in this study: the Langmuir probe (LAP) (Eriksson et al., 2007), the fluxgate magnetometer (MAG) (Glassmeier et al., 2007b) and the electrostatic ion and electron sensor (IES) (Burch et al., 2007), all interfacing to the spacecraft through the plasma interface unit (PIU) (Carr et al., 2007). The Rosetta LAP instrument measured probe to spacecraft potential during the Mars flyby from the one probe out of two that was located in sunlight upstream of the spacecraft. Rosetta MAG measured the vector magnetic field with a time resolution of $1 \mathrm{~Hz}$. Rosetta IES has a time resolution of $128 \mathrm{~s}$ and measures electrons in the energy range $0.001-22 \mathrm{keV} / \mathrm{q}$ with a field of view of $90^{\circ} \times 360^{\circ}$. MEX carries the Mars advanced radar for subsurface and ionospheric sounding (MARSIS) instrument (Picardi et al., 2004) and the analyzer of space plasma and energetic atoms (ASPERA-3) instrument, which includes the electron spectrometer (ELS) and the ion mass analyzer (IMA) (Barabash et al., 2006). IMA measures ions in the energy range 0.01$36 \mathrm{keV} / \mathrm{q}$ with a time resolution of $192 \mathrm{~s}$ and a field of view of $90^{\circ} \times 360^{\circ}$. ELS measures electrons in the energy range $0.001-20 \mathrm{keV} / \mathrm{q}$ (electrons with energies below $10 \mathrm{eV}$ are however affected by the spacecraft potential and are not always reliable) with a time resolution of $4 \mathrm{~s}$ and a field of view of $4^{\circ} \times 360^{\circ}$. MARSIS is designed for ionospheric and subsurface sounding but can occasionally also measure local plasma density and magnetic field magnitude from observed harmonics of the electron plasma oscillations and electron cyclotron echoes in the radargrams, respectively (Gurnett et al., 2005; Duru et al., 2008).

The orbit geometry of MEX and Rosetta at the time of the Rosetta Mars swingby is shown in Fig. 1. Also shown are the positions of the MPB and BS crossings observed by MEX from 00:00 UT on 24 February 2007 until 24:00 UT on 27 February 2007 together with empirical models of the two boundaries from Edberg et al. (2008) and Trotignon et al. (2006). The position of each crossing is adjusted to account for the mean $4^{\circ}$ aberration of the solar wind direction caused by the orbital motion of the planet while the MEX orbit (black solid line) is not adjusted. Note that the inbound and the outbound boundary crossings occurred on almost opposite sides of the planet. MEX was at this time in an elliptical 6-h orbit very close to the terminator plane of Mars with periapsis at about $310 \mathrm{~km}$ and apoapsis at about $10000 \mathrm{~km}$. It hence crossed the MPB and the BS both inbound and 

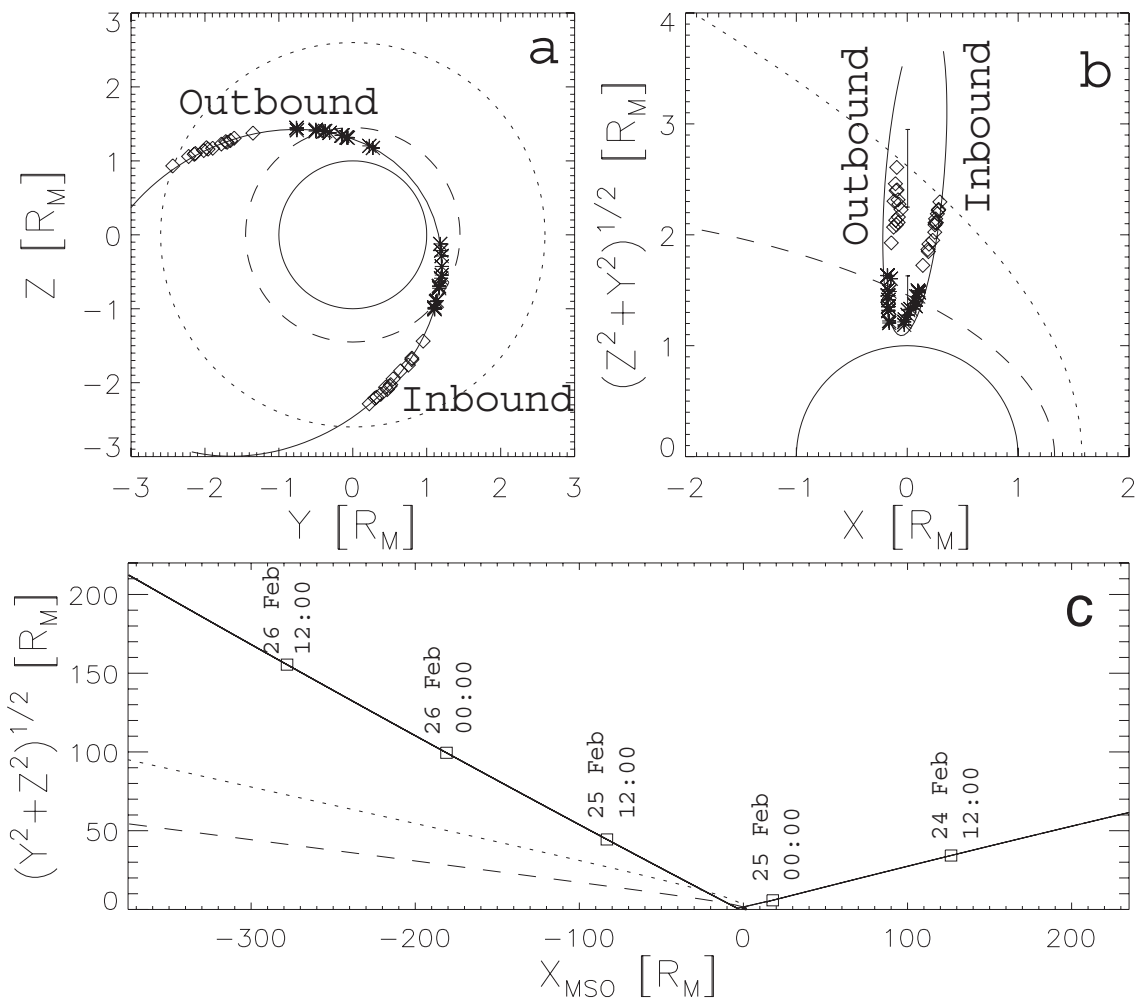

Fig. 1. Boundary crossing locations and orbital geometry during the Rosetta Mars swingby. The panels show all BS (diamonds) and MPB (stars) crossings observed by MEX during 24-27 February 2007, projected onto (a) the y-z plane and (b) in cylindrical coordinates together with the average BS (dotted line) and MPB (dashed line) as well as (c) the trajectory of Rosetta in cylindrical coordinates (black solid line) together with best fits of the MPB (dashed line) and BS (dotted line) from Trotignon et al. (2006). A part of the MEX orbit is shown in panels (a) and (b) (black line). The vertical bars in panel (b) indicate the mean extrapolated terminator distance and standard deviation of the BS and MPB crossings from Edberg et al. (2008). The positions of the crossings are adjusted to account for the $4^{\circ}$ aberrated solar wind flow caused by the orbital motion of the planet with respect to an average solar wind speed of $400 \mathrm{~km} \mathrm{~s}^{-1}$.

outbound on every orbit and completed 14 orbits during these four days. The BS, on inbound passes, is identified in the MEX/ELS data as a sudden increase in electron fluxes and the MPB as a sudden decrease in electron fluxes in the energy range $\sim 10-500 \mathrm{eV}$. In panel (c) we show the trajectory of Rosetta, which had its closest approach to Mars at 01:58 UT on 25 February 2007 and then proceeded out through the magnetosheath on the nightside, crossed the BS outbound at 04:04 UT and continued downstream in the solar wind.

\subsection{Boundary asymmetries during high pressure solar wind}

During the time of the Rosetta flyby, a high pressure solar wind region was observed by both MEX and Rosetta. This can be seen in Fig. 2 where we show a time series of Rosetta IES/LAP/MAG and MEX ELS/IMA data for the two day interval around the time of the Rosetta swingby, starting at 00:00 UT on 25 February and lasting to 24:00 UT on 26 February 2007. The Rosetta LAP probe-to-spacecraft potential $V_{\mathrm{ps}}$ can be used as a proxy for the plasma density for time periods when the spacecraft attitude, or the sun-aspect angle (SAA), is constant. The SAA is the angle about the axis through the solar panels of Rosetta and is important for determining the pointing of IES and also for the location of the LAP instrument in the potential field of Rosetta. For the range of values of probe-to-spacecraft potential and SAA presented in this paper there is roughly a linear relation with the plasma density (Edberg et al., 2009b). An IES mode change at 05:00 UT on 25 February caused electrons below $500 \mathrm{eV}$ not to be sampled afterwards. An unfavorable spacecraft attitude also affected the IES measurements with lower fluxes as a result.

At 11:49 UT on 25 February 2007, indicated by the left red vertical line in Fig. 2, IMA onboard MEX observed a sudden solar wind density increase from $\sim 1 \mathrm{~cm}^{-3}$ to a maximum of $\sim 10 \mathrm{~cm}^{-3}$ (Fig. $2 \mathrm{~g}$ ). The MEX ELS instrument also observed an increase in electron fluxes at the same time. This was followed by a gradual increase in velocity from $\sim 350 \mathrm{~km} \mathrm{~s}^{-1}$ to a maximum of $\sim 500 \mathrm{~km} \mathrm{~s}^{-1}$. For details regarding the moment calculation from IMA, see Fränz et al. 


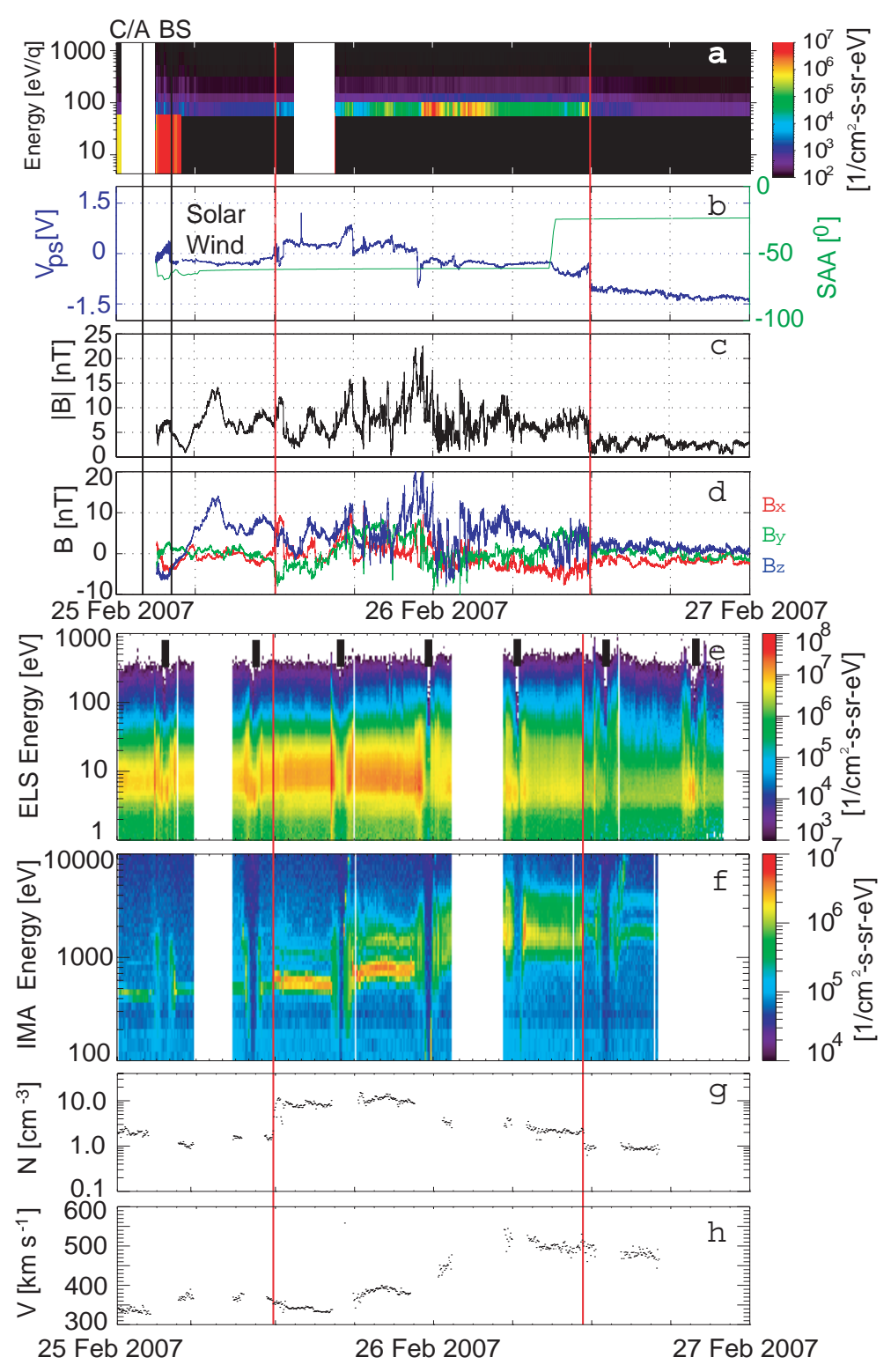

Fig. 2. Time series of (a) Rosetta IES omni-directional electron spectra, (b) Rosetta LAP probe-to-spacecraft potential $V_{\mathrm{ps}}$ measured at a rate of $0.9 \mathrm{~Hz}$ (blue) and Rosetta SAA (green), Rosetta MAG (c) magnetic field magnitude and (d) components in MSO coordinates, (e) MEX ELS omni-directional electron energy spectra, (f) MEX IMA omni-directional ion energy spectra as well as (g) MEX IMA ion density and (h) total velocity. The interval of high pressure is marked by red lines, while the closest approach of Rosetta and the outbound BS crossing is marked by black lines. The thick bars in panel (e) mark the closest approach of MEX during each orbit.

(2006). At 12:02 UT on 25 February 2007 the IES, LAP and MAG on Rosetta observed an increase in electron flux in the energy range $60-100 \mathrm{eV}$, a sharp increase in $V_{\mathrm{ps}}$, and an increase in magnetic field variability, respectively. Rosetta was downstream of Mars at a distance of $83 R_{\mathrm{M}}$ at this time. The spatial difference between where Rosetta starts to observe the high pressure solar wind and where MEX observes it divided by the difference in arrival times at the two spacecraft gives a velocity in the $-x$-direction of $\sim 360 \mathrm{~km} \mathrm{~s}^{-1}$ ( $83 R_{\mathrm{M}}$ divided by $13 \mathrm{~min}$ ).
The density increase observed by MEX lasted until 11:22 UT on 26 February 2007, indicated by the right red vertical line in Fig. 2, while the higher velocity lasted longer, at least until a data gap started on 28 February 2007. At Rosetta, $V_{\text {ps }}$ dropped suddenly (corresponding to a decrease in plasma density) at the same time as the magnetic field variability signatures stopped and the electron fluxes decreased at 11:53 UT on 26 February 2007 at a distance of $277 R_{\mathrm{M}}$ downstream of Mars. The difference in time between these signatures in the data of the two spacecraft give a velocity 


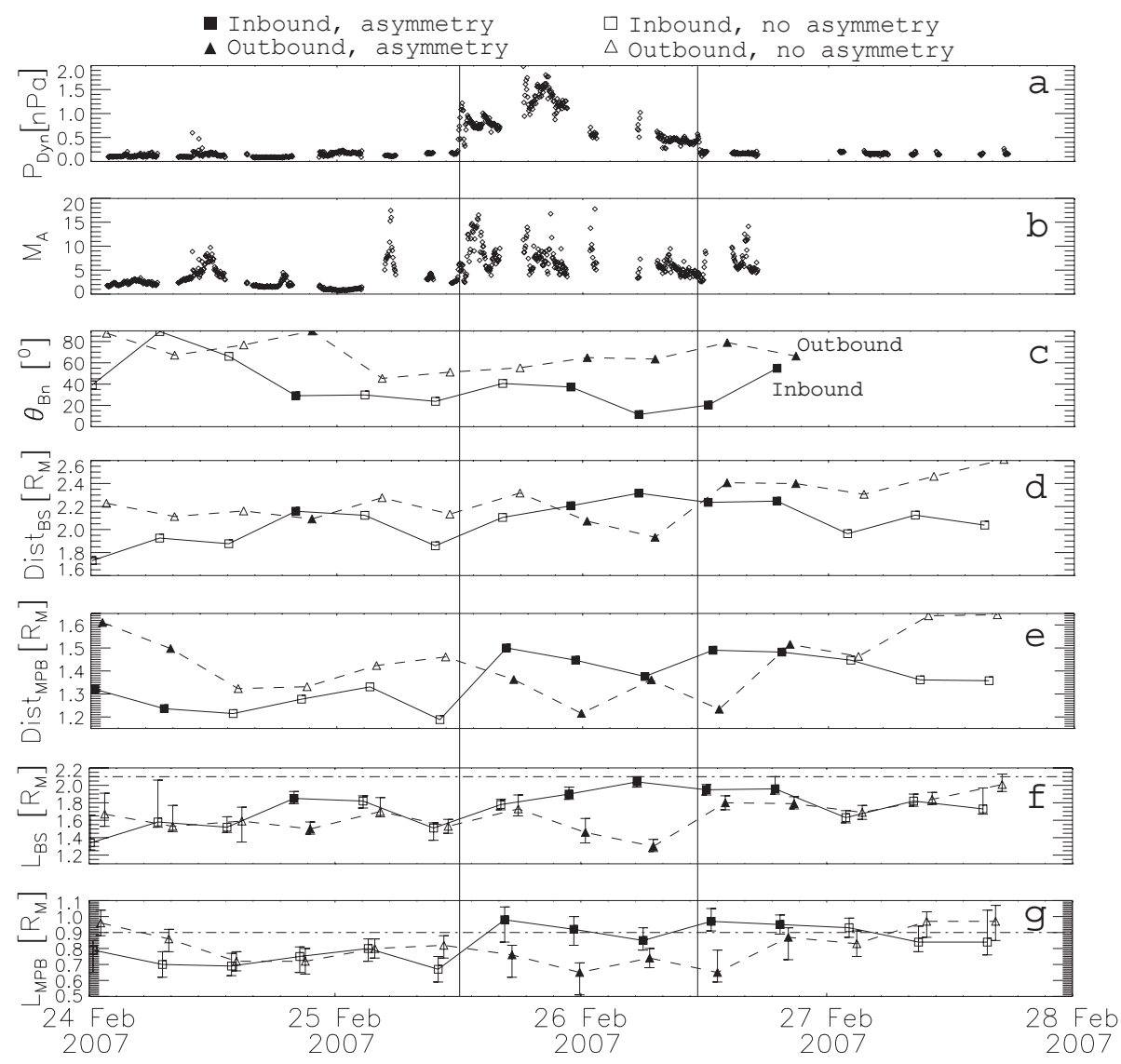

Fig. 3. Time series of (a) the MEX IMA dynamic pressure, (b) Rosetta MAG/MEX IMA Alfvénic Mach number, (c) $\theta_{\mathrm{Bn}}$, distance of each (d) BS and (e) MPB from the center of the planet as well as L-value of each (f) BS and (g) MPB together with error bars. Inbound $\theta_{\mathrm{Bn}}$, distances and L-values are displayed as squares and connected by solid lines while the outbound ones are displayed as triangles and connected by dashed lines. Filled symbols indicate that there is an asymmetry present between the inbound and outbound boundaries. The values of $L$ from Edberg et al. (2008) are indicated by dash-dotted lines in panels (f) and (g). The black vertical lines mark the interval of the high pressure solar wind.

in the $-\mathrm{x}$-direction of $\sim 510 \mathrm{~km} \mathrm{~s}^{-1}$. The solar wind velocity measured by MEX IMA agrees very well with the velocities derived from arrival times of similar features at the two spacecraft $\left(\sim 350 \mathrm{~km} \mathrm{~s}^{-1}\right.$ compared to $\sim 360 \mathrm{~km} \mathrm{~s}^{-1}$ and $\sim 510 \mathrm{~km} \mathrm{~s}^{-1}$ compared to $\sim 500 \mathrm{~km} \mathrm{~s}^{-1}$ ). The velocity does not change across the boundaries between regions of high and low pressure regions, as would be expected from interplanetary shocks or corotating interaction regions, and they rather resemble convected structures propagating outward in the solar system. This observed high pressure solar wind region and its effect on the Martian plasma environment is the topic in this paper.

To illustrate how the BS and MPB respond to the high pressure solar wind region, we plot their altitudes as a time series together with the MEX and Rosetta measurements in Fig. 3. The time series extends across four days of combined Rosetta and MEX measurements starting at 00:00 UT on 24 February 2007. In panel (a) we show solar wind dy- namic pressure derived from MEX IMA and in panel (b) the Alfvénic Mach number $M_{\mathrm{A}}$ based on a combination of the magnetic field strength from Rosetta MAG, time-shifted to the time frame of MEX assuming a constant solar wind velocity of $350 \mathrm{~km} \mathrm{~s}^{-1}$, and the solar wind plasma density from MEX IMA. Panel (c) shows the angle between the IMF, as measured by Rosetta and time-shifted to the time frame of MEX, and the local normal to the BS $\theta_{\mathrm{Bn}}$, for each inbound (squares) and outbound (triangles) BS crossing by MEX. The local normal to the BS was calculated from the statistical best fit shape of the BS from Edberg et al. (2008) at the point closest to the observed BS crossing. Inbound and outbound crossing data are displayed as squares and triangles, respectively, throughout the paper. Panel (d) shows the distance of each BS crossing from the center of the planet, for inbound and outbound crossings separately and panel (e) shows the distance of the MPB crossings. 
The inbound crossings occur at lower solar zenith angles (SZA) than the outbound ones and should therefore be at lower distances but this is evidently not always the case. The inbound crossings are sometimes at larger distances than the outbound crossing during the same orbit. Hence the boundaries are asymmetric in their shapes. In order to properly compare the distances of inbound crossings with the distance of the outbound crossings, which occur at different SZAs, we need a measure of their distances which is independent of the SZA at which they occur. Panel (f) therefore shows the value of the semi-latus rectum $L$ from fits of conic sections to each single BS crossing. The fits are done in the same way as described in Crider et al. (2002), where the values of $\epsilon$ and $X_{0}$ (from Edberg et al., 2008) are fixed, and only $L$ is varied. $L$ is hence a linear measure of the distance of a crossing for which the SZA dependence has been removed. Panel (g) shows the same as panel (f) but for the MPB instead of the BS. Due to the orbit configuration, upstream waves and also the movement of the boundaries, the two boundaries do not always appear as sharp boundaries but are at times more or less smeared out in a time series. This gives an uncertainty to where the boundary crossings actually occur. The uncertainty bars in panels (f) and (g) represent where the spacecraft is well within/without the BS and the MPB, as seen in a time series of data, and are hence estimates of the thicknesses of the boundaries, or their variability in position during each crossing.

The position of each crossing is adjusted to account for the $4^{\circ}$ aberration caused by the planets orbital velocity. The average values of $L$ for the BS and MPB from Edberg et al. (2008) are shown for reference purposes by the horizontal dash-dotted lines in panels (d) and (e). The inbound and outbound crossings of both the BS and the MPB are at approximately similar altitudes (L-values) up until the high pressure solar wind appears. They are also closer than average to the planet in the time interval preceding the high pressure solar wind. During and after the high pressure solar wind the altitude of the inbound and outbound crossings deviate significantly from each other, by up to $0.7 R_{\mathrm{M}}$ for the BS and $0.4 R_{\mathrm{M}}$ for the MPB and hence properly indicate that both boundaries have become asymmetric. The inbound boundaries move outward first and the outbound boundaries follow later on.

The asymmetry of the MPB starts during the same orbit as the high pressure solar wind feature is observed and the BS asymmetry starts during the following orbit. The shape asymmetry lasts until about two orbits after the solar wind feature has passed. As reported by Pérez-de-Tejada et al. (2009), the exosphere of Mars, as seen in X-ray images from the XMM/Newton satellite, is also very asymmetric during this interval. The X-ray emissions from the exosphere are more intense over the poles and also indicate that the exosphere over the poles is tilted in an anti-sunward direction. Note also that there is a short-lived BS asymmetry during the last orbit on 24 February 2007. Throughout the paper, filled symbols indicate that there is an asymmetry present between the inbound and outbound crossings.

\subsection{Cause of the asymmetries}

Factors that could be responsible for the asymmetry include whether the boundaries are quasi-perpendicular or quasiparallel, the direction of the solar wind convective electric field $\boldsymbol{E}=-\boldsymbol{v} \times \boldsymbol{B}$ and the crustal magnetic fields and.

1. The quasi-parallel vs quasi-perpendicular effect causes increased/decreased upstream wave activity which increases/decreases the apparent thickness of the boundaries. This has already been accounted for to some extent by including the error bars in Fig. 3 but it is possible that the boundaries also move outward or inward as a whole due to this. The MPB has not previously been shown to be affected by the quasi-parallel or quasiperpendicular direction of the IMF and for the sake of completeness we do check whether or not the MPB is affected.

2. The convective electric fields determines in which direction the ions and electrons move. On one side of the planet the ions will move into the planet and on the opposite side they will move out into the solar wind and cause increased massloading which could cause the BS to move outward due to the increased obstacle size. This is mainly expected to influence the BS and the magnetosheath, where the plasma is in motion. The convective electric field does not exist deep beneath the MPB where the plasma is not in motion. However, if ions are transported away from the magnetosheath to a larger extent, the magnetosheath plasma pressure might decrease to such an extent that the MPB can move upward.

3. The crustal magnetic fields provide additional magnetic pressure which disturbs the pressure balance across the boundaries and would cause the boundaries to move outward. Since the crustal magnetic fields are strongest and most frequently observed at southern latitudes the boundaries should be at higher altitudes over the Southern Hemisphere.

In Fig. 4 we show the results of tests of the above factors that could possibly cause the asymmetry. In panels (a) and (b) we show the value of the semi-latus rectum $L$ for each observed BS and MPB crossing, respectively, as a function of $\theta_{\mathrm{Bn}}$ together with linear least-square fits to the inbound (solid line) and outbound (dashed line) crossings. The difference in mean value of the semi-latus rectum of all quasiparallel $\left(\theta_{\mathrm{Bn}}<45^{\circ}\right)$ and all quasi-perpendicular $\left(\theta_{\mathrm{Bn}}>45^{\circ}\right)$ BS crossings (1.77 and 1.62, respectively) is statistically significant according to a Student's t-test at a $90 \%$ confidence level. However, if a Student's t-test is done separately for inbound and outbound BS crossings, then only the inbound crossings show a significant difference in mean values of $L$ 

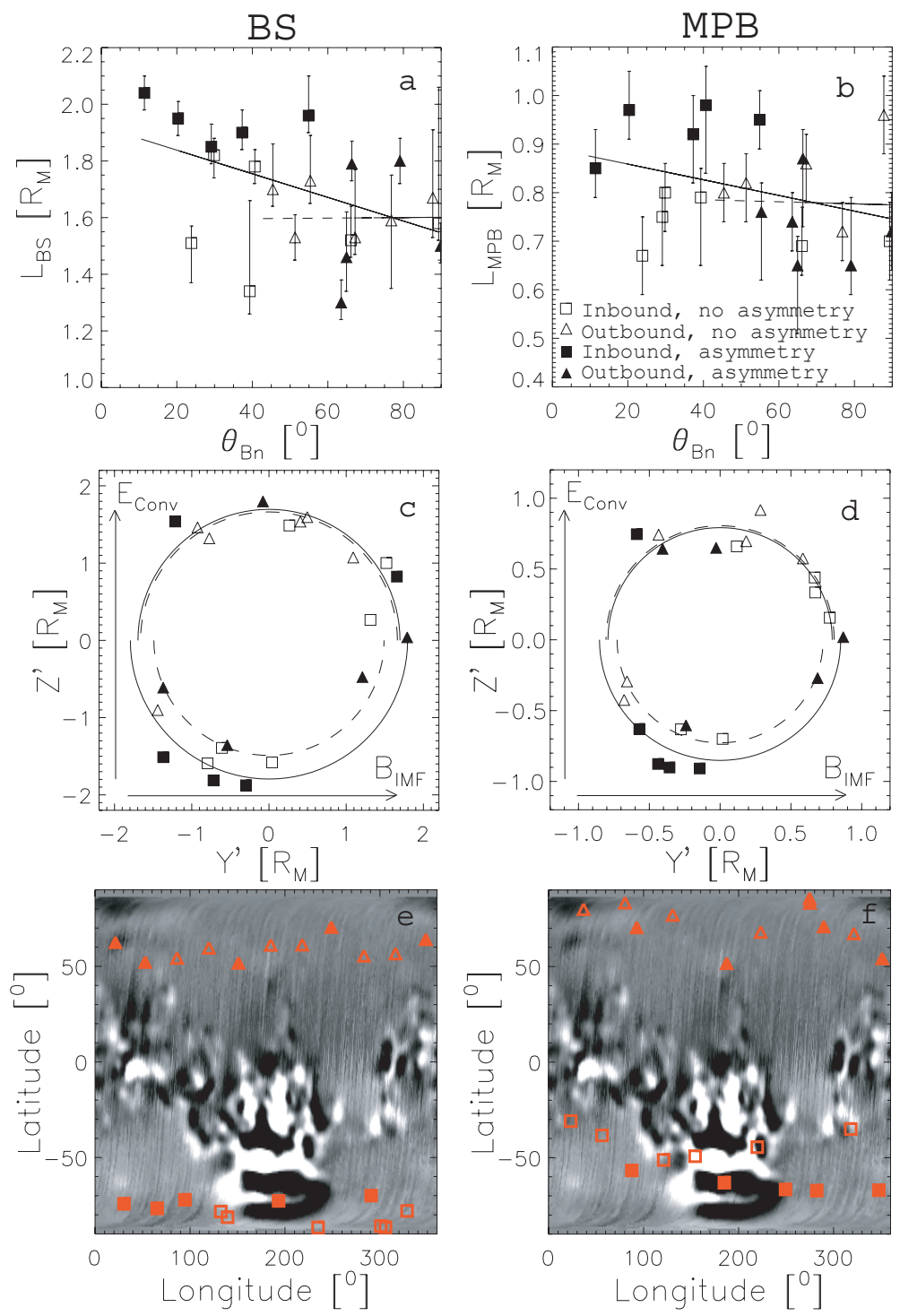

Fig. 4. The distance displayed in terms of L-value (see text for explanation) of (a) the BS and (b) the MPB crossings as a function of $\theta_{\mathrm{Bn}}$ with linear fits to the inbound (solid) and the outbound (open) crossings. The position (c) of all BS and (d) MPB crossings rotated into a frame where $Y^{\prime}$ is aligned with the IMF and $Z^{\prime}$ with the convection electric field. Average distances are shown for inbound (solid line) and outbound (dashed line) crossings in the $+E$ and $-E$ hemispheres in panels (c) and (d). The position (e) of all BS and (f) MPB crossings projected onto a map of the crustal magnetic field strength (the position data being colored red in order to make them more visible).

at a confidence level of $90 \%$. An equally significant difference between mean values of the semi-latus rectum is not found for the MPB. There is a trend of a closer in boundary for higher $\theta_{\mathrm{Bn}}$ in panel (a) and panel (b), but only for the crossings that occur when the boundary is asymmetric and not for the other crossings. This rather tells us that the crossings that occur during the asymmetric boundary interval and are on the inbound side also happens to be quasi-parallel and vice versa but not that it is the $\theta_{\mathrm{Bn}}$ angle that determines the altitude of the boundaries. Zhang et al. (1991) showed that the BS altitude at both Mars and Venus was higher in the quasi-perpendicular case than in the quasi-parallel case. In that study, a larger data set was used such that the results should be more reliable.

In panels (c) and (d) of Fig. 4 we show all BS and MPB crossings rotated into a reference frame where $Y^{\prime}$ is aligned with the instantaneous IMF direction, as measured by Rosetta MAG, and $Z^{\prime}$ with the convection electric field direction, in order to determine the effect of the convection electric field. Average distances are displayed for inbound (solid) and outbound (dashed line) crossings in both the $+E$-hemisphere, where the electric field is directed 
locally upward, and in the $-E$ hemisphere, where the electric field is directed locally downward. If asymmetric massloading occurred and was the main factor which controlled the location of the boundaries, then the boundaries in the $+E$-hemisphere, where the ions move out into the solar wind, would be at higher altitudes than the ones in the $-E$ hemisphere, where the ions move toward the planet. However, no such trend is observed for either the BS or the MPB, neither during high nor low pressure periods.

We do, however, observe that in the $-E$-hemisphere the inbound crossings, in the Southern/dusk Hemisphere, are further out than the outbound crossings. The difference is statistically significant according to Student's t-test at a 95\% confidence level for the BS and at a $90 \%$ confidence level for the MPB. This asymmetry is not visible for the $+E$-hemisphere. Since the evolution of the IMF orientation with distance is unknown, there could be a difference in IMF orientation between the location where Rosetta measures the magnetic field and the location where MEX measures the plasma boundaries.

In panels (e) and (f) of Fig. 4 we show, respectively, the BS and MPB crossings projected on a map of the crustal magnetic field strength to see if the observed asymmetry is caused by the fact that some crossings occur over strong crustal fields and others not. However, the asymmetric boundary crossings occur over a wide range of longitudes and the inbound crossings, at southern latitudes, do not occur directly over the strong crustal fields, which could have caused them to move to higher altitudes by providing additional magnetic pressure. The crustal magnetic fields do not therefore seem to be responsible for causing an asymmetry in this case. In fact, neither of the factors tested in Fig. 4 give a clear result as to whether they affect the location of the boundaries to become asymmetric or not.

\subsection{Ion outflow and exosphere asymmetry}

Figure 5 shows MEX measurements from three consecutive orbits beginning on 25 February 2007 which occur during equally long intervals centered at the time of the MEX closest approach to Mars on each pass. In panels (a), (d) and (g) we show IMA omni-directional ion energy spectrograms of heavy planetary ions together with MEX altitude (red line), in panel (b), (e) and (h) we show ELS omni-directional electron energy spectrograms together with MARSIS measured local electron density (short black line between $30 \mathrm{~cm}^{-3}$ and $70 \mathrm{~cm}^{-3}$ ) and in panels (c), (f) and (i) we show Rosetta MAG magnetic field measurements time-shifted to the time frame of MEX. The time-shifts are $\sim 11 \mathrm{~min}, \sim 20 \mathrm{~min}$ and $\sim 29 \mathrm{~min}$ for each orbit, respectively, if using the measured solar wind velocity of $350 \mathrm{~km} \mathrm{~s}^{-1}$ from Fig. 2, panel (h). If assuming a $\sim 10 \%$ error on the measured velocity the error on the timeshifts are $\pm 1-2 \mathrm{~min}$. There are only MARSIS density measurements during $\sim 5 \mathrm{~min}$ on the outbound part of the first orbit, $\sim 5$ min during both the inbound and the outbound part of the second orbit and only $\sim 5$ min during the inbound part of the third orbit, but these are still important since they provide a measure of the local plasma density. IMA and ELS can also provide values of the density but, unlike MARSIS, do not always measure the full population, especially not the colder population.

Panels (d) and (e) of Fig. 5 show the same data as presented by Pérez-de-Tejada et al. (2009) (their Fig. 2). From 17:10 until 17:40 UT in panel (d) a distinct population of heavy ions (mainly $\mathrm{CO}_{2}^{+}, \mathrm{O}_{2}^{+}$and $\mathrm{O}^{+}$) are seen in the energy range $100 \mathrm{eV}-10 \mathrm{keV}$, around the time of the outbound MPB crossing, which are interpreted as being heavy planetary ions escaping from the planet. These ions show a clear trend of increasing in energy as MEX measures them at higher and higher altitudes and they reach velocities far higher than the required escape velocity (Pérez-de-Tejada et al., 2009).

Not reported by Pérez-de-Tejada et al. (2009) is that such escaping planetary ions are also visible on the following orbit, in almost exactly the same location but with an order of magnitude less intensity. These are seen in panel $(\mathrm{g})$ from 23:55 UT on 25 February until 00:15 UT on 26 February, again as a distinct population which gain in energy, from $100 \mathrm{eV}$ up to $1 \mathrm{keV}$, while MEX measures them at higher altitude. The population of escaping ions during this orbit is now harder to distinguish from the background measurements but still visible. It should be noted that ions are constantly escaping from Mars, but during the interval studied in this paper there seems to be a significant increase of such escaping ions.

It is during these two orbits, when escaping planetary ions are observed, that the solar wind pressure increases to high values of $\sim 1.0-1.5 \mathrm{nPa}$ as can be seen in panel (a) of Fig. 3 . The magnetic field measurements show that the IMF was mainly northward during these orbits which gives a convective electric field direction mainly in the MSO $-\mathrm{y}$-direction. The convective electric field is in the same direction, roughly, as MEX moves in when the escaping planetary ions are observed, see MEX trajectory in Fig. 1, panel (a). As the ions are observed to gain in energy we can conclude that they are accelerated by the convective electric field. At the same time as the magnetic field changes its orientation at 17:40 UT (panel b) the escaping ions cease being observed in the ion spectrogram. Pérez-de-Tejada et al. (2009) also noted this sharp drop but suggested that it was caused by momentum transport between the planetary ions and the solar wind ions. But with the magnetic field measurements from Rosetta we now suggest that the sharp drop is caused by a change of direction of the convection electric field, rotating from $-y$ to $+z$, such that the outflowing ions start to move northward while MEX moves farther westward and ceases to observe the outflowing ions. During the second orbit when these ions are observed, the IMF direction is still mainly northward but more variable which could explain why the measured flux rates are lower than during the previous orbit.

The local electron densities measured by MARSIS just inside the MPB on the first orbit in Fig. 5, panel (b), are 

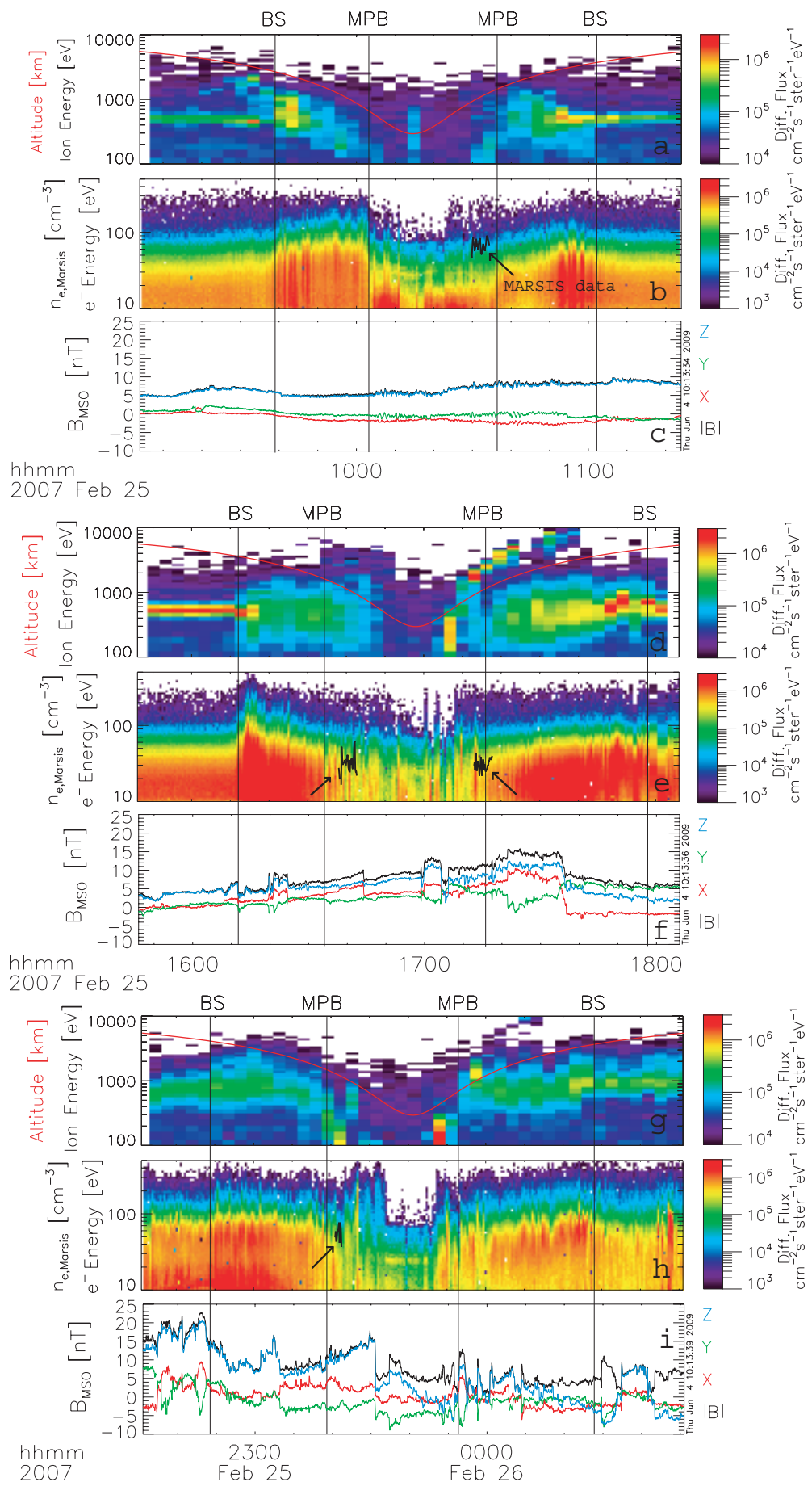

Fig. 5. Time series of MEX and Rosetta measurements. The panels show (a, $\mathbf{d}$ and $\mathbf{g}$ ) MEX heavy planetary ion omni-directional spectrograms, (b, e and $\mathbf{h}$ ) MEX electron omni-directional spectrograms and (c, $\mathbf{f}$ and $\mathbf{i}$ ) Rosetta magnetic field measurements during three consecutive MEX orbits. The downstream Rosetta measurements are time-shifted to the time frame of MEX. The heavy ion spectrograms contains a significant amount of 'ghost particles in the magnetosheath which are caused by a contamination inside the instrument by solar wind protons. The altitude of MEX is superposed on the ion spectrograms (red line, same scale as for the ion energy) and the local electron density from MARSIS is superposed on the electron spectrograms (short black line, same scale as for the electron density). The MARSIS data is only available during $\sim 5$ min on each orbit an pointed out by black arrows. The time of the BS and MPB crossings by MEX are shown as vertical black lines. 
$\sim 70 \mathrm{~cm}^{-3}$. During the following orbit, in panel (d), at the same altitudes on the outbound pass as in the previous orbit, the density had dropped to values of $\sim 30 \mathrm{~cm}^{-3}$. Also during the inbound pass of the second orbit the densities, at approximately the same altitudes, vary around $30 \mathrm{~cm}^{-3}$. During the third orbit, on the inbound leg in panel $(\mathrm{g})$, the average density had increased to around $70 \mathrm{~cm}^{-3}$ again. These limited measurements from MARSIS indicate, in the same way as shown by Dubinin et al. (2009) but for a different interval, that the ionospheric densities inside the MPB decreased when the high pressure solar wind impacted Mars and the ionosphere was being scavenged.

As shown in Fig. 3, the boundaries start to become asymmetric during this interval. This can be clearly seen in Fig. 5 as well. The inbound BS moves to increasingly higher altitudes during the three orbits shown, from an L-value of $1.51 R_{\mathrm{M}}$ to $1.78 R_{\mathrm{M}}$ to $1.90 R_{\mathrm{M}}$, even though the dynamic pressure increases and should push the boundary closer in. The inbound MPB follows the same trend roughly, or at least within the error bars, and moves from an L-value of $0.70 R_{\mathrm{M}}$ to $0.98 R_{\mathrm{M}}$ to $0.92 R_{\mathrm{M}}$. The outbound MPB does, however, move inward during these three orbits, from $0.82 R_{\mathrm{M}}$ to $0.76 R_{\mathrm{M}}$ to $0.65 R_{\mathrm{M}}$. The outbound BS first moves outward, from $1.53 R_{\mathrm{M}}$ to $1.73 R_{\mathrm{M}}$ like the inbound $\mathrm{BS}$, but only to move inward again during the third orbit to $1.46 R_{\mathrm{M}}$, when the solar wind dynamic pressure decreases. These data clearly show that the dynamic pressure is not always the only important factor in determining the location of the plasma boundaries.

\section{Discussion}

Below, we discuss the observations of the asymmetric boundaries by discussing the various factors involved. According to Fig. 4, panels (a) and (b), the asymmetry is generally only present when the inbound crossings are quasi-parallel and the outbound crossings are quasi-perpendicular, which is different from previous observations. We cannot, however, conclude that the effect of the difference between quasiparallel and quasi-perpendicular crossings is the only reason for the asymmetry. We can only conclude that when there is an asymmetry present, the inbound and outbound crossings have significantly different values of $\theta_{\mathrm{Bn}}$. The BS is expected, based on previous studies by e.g. Zhang et al. (1991), to be at higher altitudes in the quasi-perpendicular case than in the quasi-perpendicular case, which is opposite to what is reported here. Other factors could still be important for causing the asymmetry and, in fact, other factors which only influence the boundary on the inbound side could cause this apparent trend of a farther out quasi-parallel BS since the trend does not exist for the outbound boundary crossings. For a larger data set the statistics will be better which will probably give a clearer result, but when looking at a shorter interval like this, the system is equally, or even more, influ- enced by other factors. The $\theta_{\mathrm{Bn}}$ effect has been suggested to be rather limited at Mars due the small obstacle shape compared to the ion-gyroradius as well as due to its extended exosphere as explained by Mazelle et al. (2004).

In Fig. 4, panels (c) and (d), we have shown that asymmetric massloading is not the single responsible factor for causing the boundary asymmetries. The boundary crossings that occur in the $+E$-hemisphere are not at higher altitudes than the crossings observed in the $-E$-hemisphere, on average. We do, however, note that the boundaries in the Southern and Northern Hemispheres of Mars react differently to different directions of the convection electric field, possibly due to the presence of the southern crustal fields or, alternatively, due to whether the boundaries are quasi-perpendicular or quasiparallel. It should be noted that there are of course errors involved when using Rosetta magnetic field direction measurements to describe the position of the boundaries. One of them being the unknown reconfiguration time of the Martian plasma environment when changes in the upstream conditions occur.

In Fig. 4, panels (e) and (f), we do not find a direct correlation between the crustal field strength at the exact position of the BS/MPB crossings and the boundary distances. However, it is in the Southern Hemisphere that the boundaries are at higher altitudes but since the MEX crossings occur at a range of longitudes it would imply a global influence of the crustal fields, if they are indeed responsible.

The Alfvénic or magnetosonic Mach number and dynamic pressure variation do not directly explain the asymmetry, either. These quantities could possibly vary in resonance with the boundary sampling rate, but this is unlikely and indeed not the case here since the solar wind parameters do not vary with the same frequency as the boundaries move up and down. A comparison between the altitudes of all crossings and the solar wind dynamic pressure and Alfvénic and magnetosonic Mach number reveals no obvious trend for the set of crossings in this interval. This could otherwise have been used to normalize the boundary altitude before examining the influence from other factors. What is also interesting and unexpected is that the BS is at lower altitudes than normal during the interval preceding the high pressure pulse (24-25 February) even though the Mach number is generally low. Edberg et al. (2009c) showed that the magnetosonic Mach number is an important factor for determining the altitude of the BS and the altitude decreases linearly with increasing Mach number.

Since none of the above factors have been clearly shown to be the single main factor that causes the asymmetry we suggest that the high pressure solar wind and the ion outflow could possibly be connected to the observed asymmetries. The ion outflow can disturb the normal balance between factors that would be expected to control the location of the boundaries, i.e. the solar wind dynamic pressure, the IMF direction, the plasma pressure, magnetic pressure and the crustal fields and we can therefore only provide a suggestion 
for a possible explanation to the observations. The MPB and BS in the Southern Hemisphere, quite counter-intuitively, move outward when the high pressure solar wind reaches Mars as can be seen in Fig. 5 and which we try to illustrate by the sketch in Fig. 6. The outward moving of the boundaries can be interpreted as the plasma pressure and/or magnetic pressure inside the MPB and BS are significantly increasing during the high pressure solar wind and more so than the solar wind dynamic pressure, resulting in an outward motion of the boundaries. The pressure on the inside could increase due to an increased massloading over a longer time period on that side of the planet in combination with increased massloading over the crustal fields on the other side, and possibly as an effect of plasma heating through reconnection events when the varying IMF reaches the crustal fields.

The convective electric field is directed toward the planet in the Southern/dusk Hemisphere and the ions consequently move in that direction. In the opposite Northern/dawn Hemisphere the convective electric field is directed away from the planet and the ions can escape the planet and the plasma pressure on the inside of the MPB and BS decreases, which leads to an eventual inward motion of the plasma boundaries. The BS during the outbound part of the orbit immediately after the high pressure region has reached Mars does, however, initially move outward, which could be an effect of massloading of the solar wind by the outflowing planetary ions. The BS therefore first moves outward but after the ionosphere has eroded during the time of at least one orbit the pressure on the inside of the BS has decreased so much that the boundaries then can move inward on that side of the planet and the boundary become asymmetric. When the high pressure region has passed the boundaries become symmetric again.

We can not conclude unambiguously that the boundaries are affected by the outflowing ions (which could imply lower plasma pressure) but rather suggest that as one possibility. Another plausible explanation could be a sheared solar wind flow associated with the high pressure region, which pushes the boundaries inward over only one hemisphere. However, the measurements of the velocity vector components are strongly dependent on the instrument field of view such that this can not always be reliably determined.

\section{Conclusions}

We have shown Rosetta and MEX measurements of a high pressure (dynamic, magnetic and thermal, as can be inferred from Fig. 2) solar wind region that passed by Mars during a $24 \mathrm{~h}$ period. At the same time as the high pressure solar wind passed by, the Martian plasma boundaries as well as the exosphere are found to become asymmetric in shape. Also, an outflow of planetary ions was observed over the northern pole of Mars in the same interval. During 4-5 orbits following the impact of the high pressure solar wind region the BS and MPB as observed inbound by MEX, in the Southern/dusk
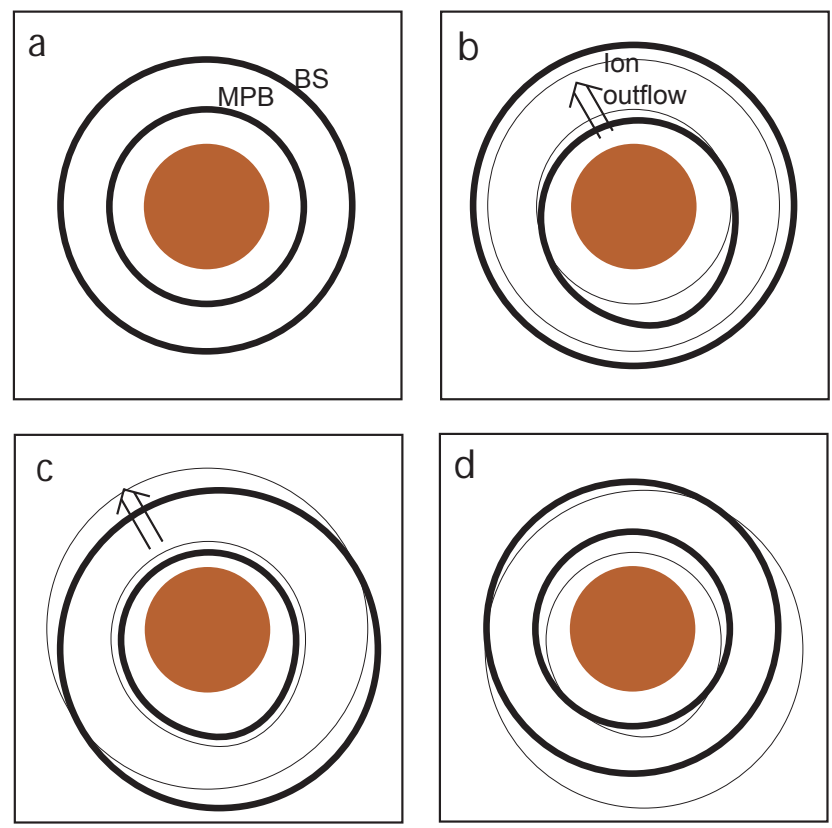

Fig. 6. Sketch of the position of the plasma boundaries in the terminator plane as seen from the Sun. In each panel are the boundaries at one specific time in the time history of the boundary positions illustrated. The movement resulting from the encounter of the high pressure solar wind as observed during the last three orbits on 25 February 2007 and when the high pressure solar wind region had passed. The thick black solid lines represent the BS and MPB, while the thin black lines in each panel represent the BS and MPB as they were in the previous panel. The boundaries go from being symmetric in panel (a) to asymmetric in panels (b) and (c) when ion outflow is observed, to finally become symmetric again in panel (d) when the high pressure solar wind has passed.

Hemisphere, were much farther out than the BS and MPB observed outbound, in the Northern/dawn Hemisphere. As reported by Dubinin et al. (2009) and Nilsson et al. (2009) the ion outflow from Mars increases during high pressure solar wind. Planetary ion outflow was, in fact, also directly observed to occur during the time of the Rosetta swingby (Pérez-de-Tejada et al., 2009). In this paper, we have combined these results and further strengthened the evidence that high pressure solar wind causes an increase of planetary ion outflow from Mars. The high pressure solar wind, observed by MAG and LAP on Rosetta and IMA and ELS on MEX, penetrated down to the ionosphere and eroded it, as observed by MARSIS on MEX, and planetary plasma was observed to escape from the planet, as observed by IMA on MEX. The solar wind plasma could also penetrate down to the ionosphere more easily during this interval since both the BS and MPB were generally located closer in than average on the orbits preceding the high pressure solar wind. The escaping ions, as observed by MEX, gained in energy in the direction, as indicated by Rosetta MAG measurements, of the 
convective electric field. The exosphere was extended over both poles and the ion outflow was observed over the Northern Hemisphere (Pérez-de-Tejada et al., 2009).

In summary, we conclude the high pressure solar wind enables the increased erosion of the ionosphere of Mars at this time. At the same as the high pressure solar wind impacts on Mars are the boundaries observed to become asymmetric in their shapes. Solar wind pressure pulses which impact on Mars seem to cause significant disturbances. They are hence important factors to consider when studying the solar wind interaction with Mars.

Acknowledgements. NJTE was supported by the European Union 6th Framework, contract MEST-CT-2004-7512. ML and SWHC were supported by STFC grant PP/E000983/1. AB was supported through the grants MO 539/13 and MO 539/15 by Deutsche Forschungsgemeinschaft. UA, KHG, and IR were supported through the grant 50 QP $9702 / 5$ of the German Ministerium für Wirtschaft und Technologie and the Deutsches Zentrum für Luftund Raumfahrt.

Topical Editor R. Nakamura thanks two anonymous referees for their help in evaluating this paper.

\section{References}

Barabash, S., Lundin, R., Andersson, H., Brinkfeldt, K., Grigoriev, A., Gunell, H., Holmström, M., Yamauchi, M., Asamura, K., Bochsler, P., Wurz, P., Cerulli-Irelli, R., Mura, A., Milillo, A., Maggi, M., Orsini, S., Coates, A. J., Linder, D. R., Kataria, D. O., Curtis, C. C., Hsieh, K. C., Sandel, B. R., Frahm, R. A., Sharber, J. R., Winningham, J. D., Grande, M., Kallio, E., Koskinen, H., Riihelä, P., Schmidt, W., Säles, T., Kozyra, J. U., Krupp, N., Woch, J., Livi, S., Luhmann, J. G., McKenna-Lawlor, S., Roelof, E. C., Williams, D. J., Sauvaud, J.-A., Fedorov, A., and Thocaven, J.-J.: The Analyzer of Space Plasmas and Energetic Atoms (ASPERA-3) for the Mars Express mission, Space Sci. Rev., 126, 113-164, doi:10.1007/s11214-006-9124-8, 2006.

Boesswetter, A., Auster, U., Richter, I., Fränz, M., Langlais, B., McKenna-Lawlor, S., Simon, S., Motschmann, U., Glassmeier, K. H., Edberg, N. J. T., and Lundin, R.: Rosetta swing-by at Mars - an analysis of the ROMAP measurements in comparison with results of 3-D multi-ion hybrid simulations and MEX/ASPERA3 data, Ann. Geophys., 27, 2383-2398, 2009, http://www.ann-geophys.net/27/2383/2009/.

Brain, D. A., Halekas, J. S., Lillis, R., Mitchell, D. L., Lin, R. P., and Crider, D. H.: Variability of the altitude of the Martian sheath, Geophys. Res. Lett., 32, L18203, doi:10.1029/2005GL023126, 2005.

Burch, J. L., Goldstein, R., Cravens, T. E., Gibson, W. C., Lundin, R. N., Pollock, C. J., Winningham, J. D., and Young, D. T.: RPC-IES: The Ion and Electron Sensor of the Rosetta Plasma Consortium, Space Sci. Rev., 128, 697-712, doi:10.1007/ s11214-006-9002-4, 2007.

Carr, C., Cupido, E., Lee, C. G. Y., Balogh, A., Beek, T., Burch, J. L., Dunford, C. N., Eriksson, A. I., Gill, R., Glassmeier, K. H., Goldstein, R., Lagoutte, D., Lundin, R., Lundin, K., Lybekk, B., Michau, J. L., Musmann, G., Nilsson, H., Pollock, C., Richter, I., and Trotignon, J. G.: RPC: The Rosetta
Plasma Consortium, Space Sci. Rev., 128, 629-647, doi:10.1007/ s11214-006-9136-4, 2007.

Crider, D. H., Acuña, M. H., Connerney, J. E. P., Vignes, D., Ness, N. F., Krymskii, A. M., Breus, T. K., Rème, H., Mazelle, C., Mitchell, D. L., Lin, R. P., Cloutier, P. A., and Winterhalter, D.: Observations of the latitude dependence of the location of the martian magnetic pileup boundary, Geophys. Res. Lett., 29, L8, doi:10.1029/2001GL013860, 2002.

Crider, D. H., Vignes, D., Krymskii, A. M., Breus, T. K., Ness, N. F., Mitchell, D. L., Slavin, J. A., and Acuña, M. H.: A proxy for determining solar wind dynamic pressure at Mars using Mars Global Surveyor data, J. Geophys. Res., 108, A12, doi:10.1029/ 2003JA009875, 2003.

Dubinin, E., Fränz, M., Woch, J., Roussos, E., Barabash, S., Lundin, R., Winningham, J. D., Frahm, R. A., and Acuña, M.: Plasma morphology at Mars: Aspera-3 observations, Space Sci. Rev., 126, 209-238, doi:10.1007/s11214-006-9039-4, 2006.

Dubinin, E., Chanteur, G., Fraenz, M., Modolo, R., Woch, J., Roussos, E., Barabash, S., Lundin, R., and Winningham, J. D.: Asymmetry of plasma fluxes at Mars. ASPERA-3 observations and hybrid simulations, Planet. Space Sci., 56, 832-835, doi: 10.1016/j.pss.2007.12.006, 2008a.

Dubinin, E., Fraenz, M., Woch, J., Duru, F., Gurnett, D., Modolo, R., Barabash, S., and Lundin, R.: Ionospheric storms on Mars: Impact of the corotating interaction region, Geophys. Res. Lett., 36, L01105, doi:10.1029/2008GL036559, 2009.

Dubinin, E. M., Fraenz, M., Woch, J., Roussos, E., Winningham, J. D., Frahm, R. A., Coates, A., Leblanc, F., Lundin, R., and Barabash, S.: Access of solar wind electrons into the Martian magnetosphere, Ann. Geophys., 26, 3511-3524, 2008b, http://www.ann-geophys.net/26/3511/2008/.

Duru, F., Gurnett, D. A., Morgan, D. D., Modolo, R., Nagy, A. F., and Najib, D.: Electron densities in the upper ionosphere of Mars from the excitation of electron plasma oscillations, J. Geophys. Res., 113, A07302, doi:10.1029/2008JA013073, 2008.

Edberg, N. J. T., Lester, M., Cowley, S. W. H., and Eriksson, A. I.: Statistical analysis of the location of the Martian magnetic pileup boundary and bow shock and the influence of crustal magnetic fields, J. Geophys. Res., 113, A08206, doi:10.1029/ 2008JA013096, 2008.

Edberg, N. J. T., Brain, D. A., Lester, M., Cowley, S. W. H., Modolo, R., Fränz, M., and Barabash, S.: Plasma boundary variability at Mars as observed by Mars Global Surveyor and Mars Express, Ann. Geophys., 27, 3537-3550, 2009a, http://www.ann-geophys.net/27/3537/2009/.

Edberg, N. J. T., Eriksson, A. I., Auster, U., Barabash, S., Bößwetter, A., Carr, C. M., Cowley, S. W. H., Cupido, E., Fränz, M., Glassmeier, K.-H., Goldstein, R., Lester, M., Lundin, R., Modolo, R., Nilsson, H., Richter, I., Samara, M., and Trotignon, J. G.: Simultaneous measurements of Martian plasma boundaries by Rosetta and Mars Express, Planet. Space Sci., 57, 1085-1096, doi:10.1016/j.pss.2008.10.016, 2009b.

Edberg, N. J. T., Lester, M., Cowley, S. W. H., Brain, D. A., Fränz, M., and Barabash, S.: Magnetosonic Mach number effect of the position of the bow shock at Mars in comparison to Venus, J. Geophys. Res., in press, 2009c.

Eriksson, A. I., Boström, R., Gill, R., Åhlén, L., Jansson, S.-E., Wahlund, J.-E., André, M., Mälkki, A., Holtet, J. A., Lybekk, B., Pedersen, A., and Blomberg, L. G.: RPC-LAP: The Rosetta 
Langmuir Probe Instrument, Space Sci. Rev., 128, 729-744, doi: 10.1007/s11214-006-9003-3, 2007.

Fränz, M., Dubinin, E., Roussos, E., Woch, J., Winningham, J. D., Frahm, R., Coates, A. J., Fedorov, A., Barabash, S., and Lundin, R.: Plasma moments in the environment of Mars, Space Sci. Rev., 126, 165-207, doi:10.1007/s11214-006-9115-9, 2006.

Glassmeier, K.-H., Boehnhardt, H., Koschny, D., Kührt, E., and Richter, I.: The Rosetta Mission: Flying Towards the Origin of the Solar System, Space Sci. Rev., 128, 1-4, doi:10.1007/ s11214-006-9140-8, 2007a.

Glassmeier, K.-H., Richter, I., Diedrich, A., Musmann, G., Auster, U., Motschmann, U., Balogh, A., Carr, C., Cupido, E., Coates, A., Rother, M., Schwingenschuh, K., Szegö, K., and Tsurutani, B.: RPC-MAG The Fluxgate Magnetometer in the ROSETTA Plasma Consortium, Space Sci. Rev., 128, 649-670, doi:10.1007/ s11214-006-9114-x, 2007b.

Gurnett, D. A., Kirchner, D. L., Huff, R. L., Morgan, D. D., Persoon, A. M., Averkamp, T. F., Duru, F., Nielsen, E., Safaeinili, A., Plaut, J. J., and Picardi, G.: Radar Soundings of the Ionosphere of Mars, Science, 310, 1929-1933, doi:10.1126/science. 1121868, 2005.

Mazelle, C., Winterhalter, D., Sauer, K., Trotignon, J. G., Acuña, M. H., Baumgärtel, K., Bertucci, C., Brain, D. A., Brecht, S. H., Delva, M., Dubinin, E., Øieroset, M., and Slavin, J.: Bow Shock and Upstream Phenomena at Mars, Space Sci. Rev., 111, 115181, doi:10.1023/B:SPAC.0000032717.98679.d0, 2004.

Nilsson, H., Carlsson, E., Brain, D. A., Yamauchi, M., Holmström, M., Barabash, S., Lundin, R., and Futaana, Y.: Ion escape from Mars as a function of solar wind conditions: A statistical study, Icarus, in press, doi:10.1016/j.icarus.2009.03.006, 2009.
Pérez-de-Tejada, H., Lundin, R., Durand-Manterola, H., and ReyesRuiz, M.: Solar wind erosion of the polar regions of the Mars ionosphere, J. Geophys. Res., 114, A02106, doi:10.1029/ 2008JA013295, 2009.

Picardi, G., Biccari, D., Seu, R., Plaut, J., Johnson, W. T. K., Jordan, R. L., Safaeinili, A., Gurnett, D. A., Huff, R., Orosei, R., Bombaci, O., Calabrese, D., and Zampolini, E.: MARSIS: Mars Advanced Radar for Subsurface and Ionosphere Sounding, in: Mars Express: the Scientific Payload, edited by Wilson, A. and Chicarro, A., vol. 1240 of ESA Special Publication, pp. 51-69, 2004.

Trotignon, J. G., Mazelle, C., Bertucci, C., and Acuña, M. H.: Martian shock and magnetic pile-up boundary positions and shapes determined from the Phobos 2 and Mars Global Surveyor data sets, Planet. Space Sci., 54, 357-369, doi:10.1016/j.pss.2006.01. 003, 2006.

Vignes, D., Mazelle, C., Reme, H., Acuña, M. H., Connerney, J. E. P., Lin, R. P., Mitchell, D. L., Cloutier, P., Crider, D. H., and Ness, N. F.: The solar wind interaction with Mars: locations and shapes of the bow shock and the magnetic pile-up boundary from the observations of the MAG/ER experiment onboard Mars Global Surveyor, Geophys. Res. Lett., 27, 49-52, doi:10.1029/1999GL010703, 2000.

Vignes, D., Acuña, M. H., Connerney, J. E. P., Crider, D. H., Rème, H., and Mazelle, C.: Factors controlling the location of the bow shock at Mars, Geophys. Res. Lett., 29, L9, doi: 10.1029/2001GL014513, 2002.

Zhang, T.-L., Schwingenschuh, K., Russell, C. T., and Luhmann, J. G.: Asymmetries in the location of the Venus and Mars bow shock, Geophys. Res. Let., 18, 127-129, doi:10.1029/ 90GL02723, 1991. 